\title{
Test of the Popular Benzodiazepine Alprazolam in a Positive Conditioned Place-Preference Model in Female Rats
}

\author{
Cheyenne Moffett ${ }^{1}$, Katherine J. Kost ${ }^{1}$, Austen Thompson ${ }^{1}$, Michael H. Ossipov ${ }^{1}$, \\ Joseph V. Pergolizzi Jr.2,3,4, Sumiyo Umeda-Raffa5, Robert B. Raffa ${ }^{3,4,6,7}$, \\ Tally M. Largent-Milnes ${ }^{1}$, Todd Vanderah ${ }^{1 *}$ \\ ${ }^{1}$ Department of Pharmacology, and Comprehensive Pain and Addiction Center, University of Arizona, Tucson, AZ, USA \\ ${ }^{2}$ NEMA Inc., Naples, FL, USA \\ ${ }^{3}$ Neumentum Inc., Morristown, NJ, USA \\ ${ }^{4}$ Enalare Therapeutics, Naples, FL, USA \\ ${ }^{5}$ Pharmaceutical Sciences (Form. Faculty), Hokkaido University of Science, Hokkaido, Japan \\ ${ }^{6}$ School of Pharmacy (Prof Em.), Temple University, Philadelphia, PA, USA \\ ${ }^{7}$ College of Pharmacy (Adjunct), University of Arizona, Tucson, AZ, USA \\ Email: *vanderah@arizona.edu
}

How to cite this paper: Moffett, C., Kost, K.J., Thompson, A., Ossipov, M.H., Pergolizzi Jr., J.V., Umeda-Raffa, S., Raffa, R.B., Largent-Milnes, T.M. and Vanderah, T. (2021) Test of the Popular Benzodiazepine Alprazolam in a Positive Conditioned Place-Preference Model in Female Rats. Pharmacology \& Pharmacy, 12, 100-108. https://doi.org/10.4236/pp.2021.125010

Received: April 27, 2021

Accepted: May 28, 2021

Published: May 31, 2021

Copyright $\odot 2021$ by author(s) and Scientific Research Publishing Inc. This work is licensed under the Creative Commons Attribution International License (CC BY 4.0).

http://creativecommons.org/licenses/by/4.0/

\begin{abstract}
The benzodiazepines were introduced into medical practice during the 1960s. At the time, they represented a safer alternative to extant therapies used for anxiety, such as the barbiturates. However, on September 23, 2020, the United States FDA indicated that more is needed to be known about the initiation, continuation, and discontinuation of the use of these widely-used drugs with publication of the announcement "to address the serious risks of abuse, addiction, physical dependence, and withdrawal reactions, the U.S. Food and Drug Administration (FDA) is requiring the Boxed Warning be updated for all benzodiazepine medicines." Because for many years, there has been a sparsity of research on these drugs, relevant information is unfortunately lacking at this critical time. It is therefore valuable to (re)establish animal models and begin to collect relevant data. We here use a model of conditioned place preference (CPP) which suggests that the representative benzodiazepine alprazolam induces positive place preference in female rats.
\end{abstract}

\section{Keywords}

Benzodiazepine, Conditioned Placepreference, Alprazolam, Female Rats

\section{Introduction}

The history of the discovery [1], development [2], clinical use [3] and problems 
[4] related to the benzodiazepines has been extensively reviewed. The benzodiazepines (Figure 1) were introduced into medical practice during the 1960s. And because of their actual, perceived, or hyped superior efficacy, tolerability, and safety profile compared to existing drug classes such as barbiturates, they rapidly became widely prescribed by physicians and requested by patients [5]. Multiple benzodiazepines are currently approved for several indications, including anxiety, insomnia, muscle relaxation, spasticity due to central nervous system pathology, and epilepsy, as well as for intra-operative use due to amnesic and ant anxiety properties [6]. Options are provided by individual differences in ADME (absorption, distribution, metabolism, and elimination). Their use increased rapidly, and they continued to be among the most commonly prescribed drugs, even more for women than for men (Figure 2) [7] [8] [9].

There was also the perception that benzodiazepines had little abuse and dependence liability. Now after decades of experience, it is clear that although benzodiazepines are safer than the barbiturates and other drugs that they largely<smiles>[R]C1=NCC(c2ccccc2[R])=NC([R])N1[R1]</smiles>

Figure 1. Benzodiazepines are a chemical family of compounds comprised of the fusion of a 6-carbon benzene ring and a 7-carbon diazepine ring. Substitutions at sites indicated by $\mathrm{R}$ result in the large number and variety of analogs.

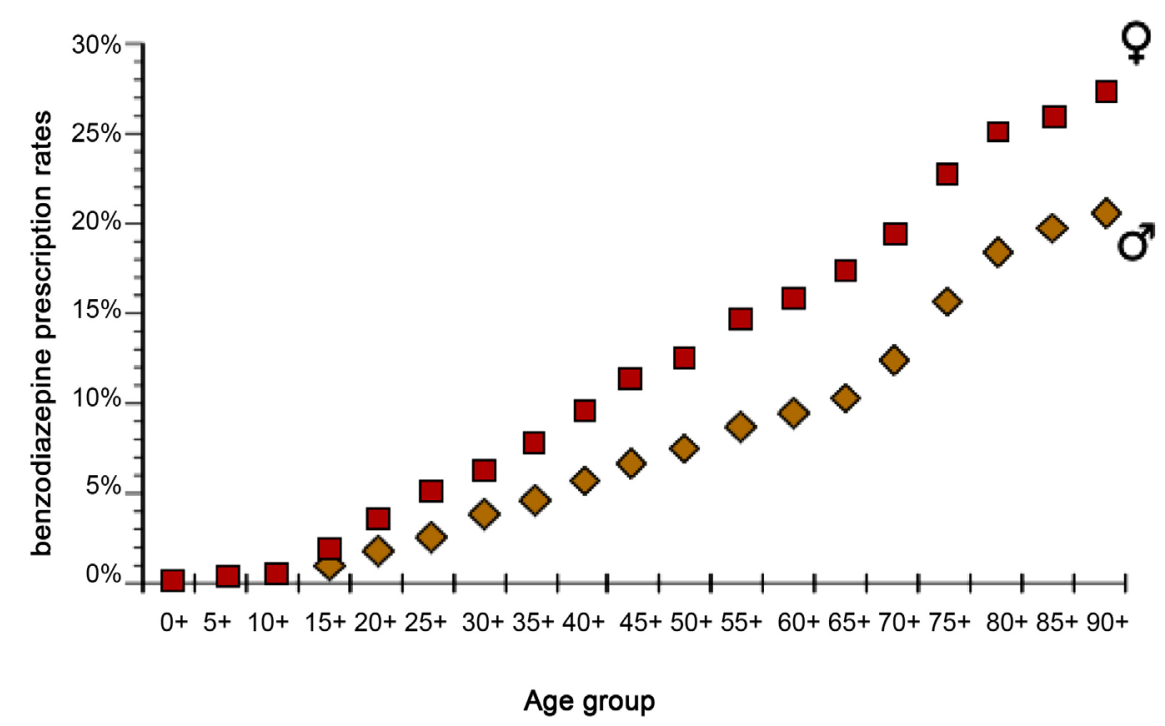

Figure 2. Male vs female benzodiazepine prescription rates ( $\mathrm{Y}$-axis) as function of age. https://www.ti.ubc.ca/2004/12/31/use-of-benzodiazepines-in-bc-is-it-consistent-with-rec ommendations/ 
replaced, they still carry some substantial risks [6]. In fact, evidence of potential abuse and dependence was reported at least as long ago as the 1980s [2] [10]. And a clear withdrawal phenomenon, sometimes extending for a prolonged period, became apparent [11]. For these reasons, on 23 September 2020, the FDA issued a required update to the boxed warning for all benzodiazepines to address the risks of abuse, misuse, addiction, physical dependence, and withdrawal reactions.

Drugs with a history of abuse potential (e.g., amphetamines, cocaine, opioids) act in part by activating reward (positive-reinforcing) centers of the brain [12]. This gives rise to a positively-reinforcing state [13]. Animal models have been developed to screen drugs for potential to induce such states in humans. These models are predicated on the observation that the relevant drug-induced state is indicated when the drug can influence, or reinforce, behavior when the behavior is associated with a rewarding stimulus [14]. One of the more commonly used animal models for the assessment of rewarding stimuli as a predictor of abuse potential is conditioned place preference (CPP) [14]. In CPP, an animal learns by exposure to associate a particular location with a rewarding stimulus. This is then revealed during testing, when the animal will spend a significantly greater amount of time in the chamber associated with the reward than in one that has not previously been associated with the reward.

The use and misuse of benzodiazepines by women is greater than is the use by men throughout the age range. [15] Whether this is due to physiological reasons (e.g., difference in prevalence of the condition, differences in genetic, epigenetic, or other differences) or to psychosocial or other reasons (e.g., healthcare access, differential prescribing biases, etc.) is not known. Whatever the reason, it is imperative that animal models reflect this difference. CPP has been used in the past to examine sex differences in dug "liking" (albeit not often) [16] [17] [18]. We are unaware of any test of female rats in CPP. In the present investigation, we demonstrate that the benzodiazepine alprazolam induces positive place preference in female rats.

\section{Methods}

Female Sprague Dawley rats (250 - 350 g) were purchased from Envigo Laboratories (Indianapolis, IN) and were maintained in a climate-controlled room on a $12 \mathrm{hr}$ light/dark cycle with food and water available ad libitum. All experiments were performed in accordance with policies and recommendations of the International Association for the Study of Pain (IASP) and the National Institutes of Health (NIH) guidelines for the handling and use of laboratory animals. The protocol and testing procedures were approved by the Institutional Animal Care and Use Committee (IACUC) of the University of Arizona. All efforts were made to avoid or minimize animal suffering and to reduce the number of animals used.

The CPP protocol was conducted as previously described [19] over a 
three-day period using test a system (San Diego Instruments) consisting of two "pairing" chambers and a smaller, well-lit "middle" chamber. The pairing chambers were dimly lit, and consisted of solid-colored walls (white) with a textured floor. The second chamber was markedly different: it had striped walls (black and white) and a smooth floor. The smaller, well-lit middle chamber was used to introduce the animal to the system, and had no artificial odor associated with it. On the first day, a rat was individually placed in the middle chamber, and had free access to all chambers over a period of 15 minutes. Automated infrared beams were used to quantify the time spent in each of the chambers. During this period, the time spent in each chamber was recorded to ensure that there were no inherent biases for one chamber over another. If a rat showed an innate bias towards one chamber (defined as spending more than $80 \%$ of the time there), it was removed from the study without further evaluation. On the second day of testing, the rats proceeded to the conditioning protocol. They were injected with alprazolam ( $0.3 \mathrm{mg} / \mathrm{kg}$, i.p., twice a day for 14 days) or vehicle (10:10:80 mixture of Tween80, DMSO, and saline) and were immediately placed into one of the two pairing chambers for $30 \mathrm{~min}$, without access to the other chambers. This was the chamber in which the rats would receive the drug injection, so was designated the "paired chamber"; the chamber not associated with drug injection was designated the "neutral chamber". The pairing for each rat was balanced, i.e., half of the group received drug (or vehicle) treatment in the solid-colored chamber, and the other half received drug (or vehicle) in the striped chamber.

Testing was performed on the following day (day 15 of the protocol), by placing each rat individually in the middle chamber and allowing it free access to roam across all chambers. The time spent in each chamber was recorded, and a difference score was derived by subtracting the time spent in the neutral chamber from the time spent in the paired chamber. In this paradigm a significant positive difference score is indicative of preference for the drug-pairing (CPP); a significant negative difference score is indicative of aversion to the drug-pairing CPA.

The mean time that the rats spent in the paired-chamber and the neutral-chamber was calculated for each group $( \pm S E M)$. Student's t-test was used to evaluate differences between the mean times for statistical significance $(\mathrm{P}>$ $0.05)$.

\section{Results}

The rats that received vehicle (10:10:80 of DMSO:Tween80:Saline) injection had a non-significant $(\mathrm{P}>0.05)$ mean difference score between chambers, indicating a lack of pre-testing preference for a chamber. Likewise, the rats treated with alprazolam had a non-significant $(\mathrm{P}>0.05)$ mean difference score between chambers, indicating the lack of a pre-testing chamber preference (Figure 3). Moreover, the mean difference scores of vehicle-treated rats and alprazolam-treated 
rats were not significantly different from each other $(P>0.05)$. More rats injected with vehicle showed a decrease in time spent in the paired chamber, compared to rats injected with alprazolam (Figure 4).

For the analyses, CPP was defined as an increase in time spent in the paired chamber of more than $50 \mathrm{sec}$, and CPA was correspondingly defined as a decrease in time spent in the paired chamber of more than $50 \mathrm{sec}$. Neutral behavior was designated when the time spent in either chamber did not differ from baseline time by more than $50 \mathrm{sec}$. Of the vehicle-treated rats, 30\% displayed CPP, $10 \%$ displayed neutral behavior, and $60 \%$ showed CPA. In contrast, $56 \%$ of the alprazolam-treated rats displayed CPP, 22\% CPA, and 22\% neutral behavior (Figure 5). The percent of animals displaying CPP was significant $(\mathrm{P} \leq 0.05)$. Likewise, the difference in percent of rats showing CPA in both treatments was significant $(\mathrm{P} \leq 0.05)$.

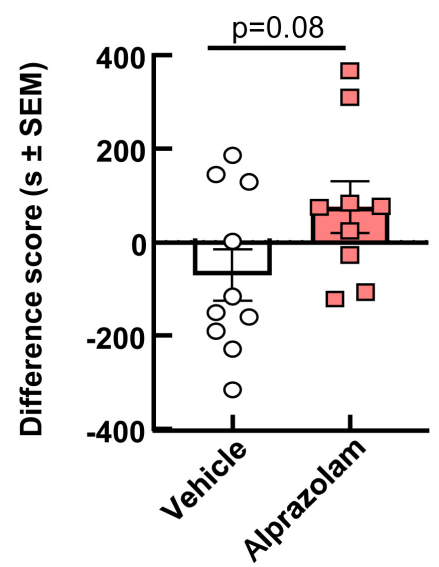

Figure 3. The mean $( \pm$ SEM) difference score (sec) between time spent in the paired and the neutral chamber for rats receiving vehicle or alprazolam (3 mg/kg, s.c.). There was no significant difference $(\mathrm{P}>0.05))$ in difference score between the vehicle-treated $(\mathrm{N}=$ $10)$ and alprazolam-treated $(\mathrm{N}=9)$ female rats.
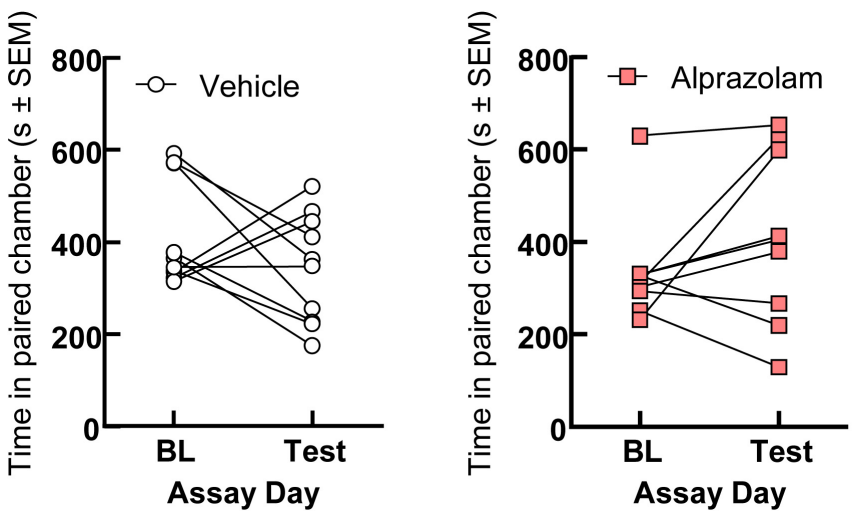

Figure 4. Time spent in the paired chamber at baseline and on test day is shown for each rat receiving vehicle $(\mathrm{N}=10)$ or alprazolam $(\mathrm{N}=9)$. More rats receiving alprazolam had increased time spent in the paired chamber compared to the vehicle-treated cohort. 
Vehicle

Total $=10$

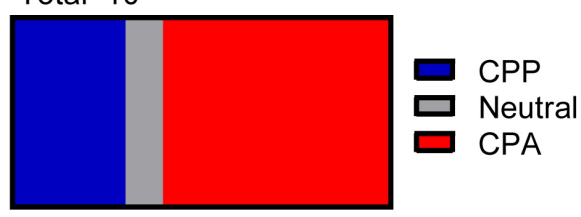

Alprazolam

Total $=9$

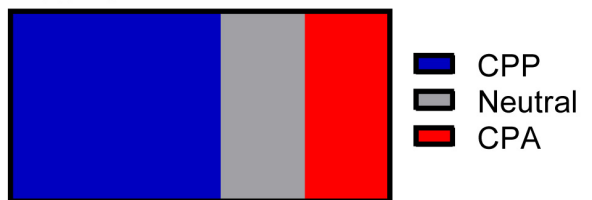

Figure 5. In vehicle-treated rats, CPP occurred in 30\% of rats and $\mathrm{CPA}$ in $60 \%$. In the alprazolam-treated group, CPP was shown in $56 \%$ of the rats and CPA in $22 \%$. The percentage of animals showing CPP was statistically significant $(\mathrm{P}<0.05)$. Likewise, the difference in percentage of animals showing $\mathrm{CPA}$ in both treatments was also statistically significant $(P<0.05)$.

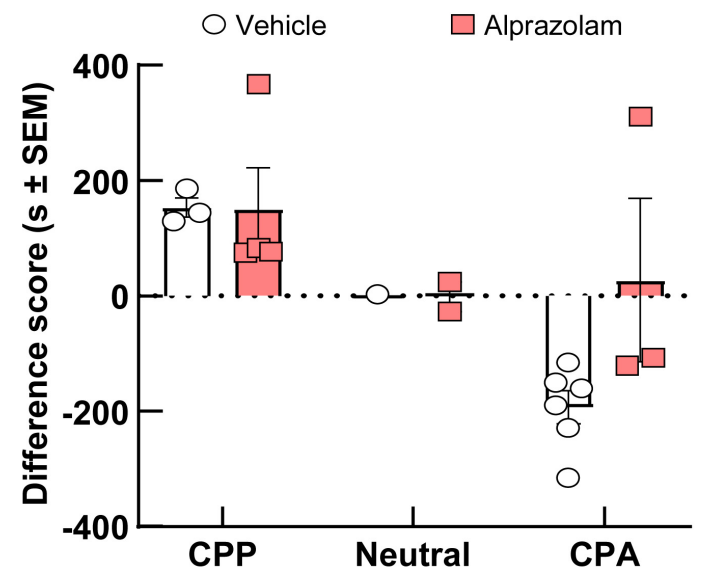

Figure 6. There were no significant differences $(\mathrm{P}>$ $0.05)$ in scores between the vehicle-treated and alprazolam-treated groups for CPP, neutral behavior, or for CPA.

The obtained difference scores were stratified by individual outcomes (i.e.; CPP, neutral behavior, and CPA) and compared to each other for both treatments by two-way ANOVA followed by Bonferroni post-hoc test for an outcome $\mathrm{x}$ treatment interaction (Figure 6). The analysis indicated that there were no significant differences between the two treatment groups in magnitude of mean difference scores for CPP, neutral behavior, or CPA (Bonferroni $\mathrm{F}_{(2,13)}=1.682$; $\mathrm{P}=0.22)$.

\section{Discussion}

Short acting benzodiazepines are highly abused medications due to their rapid 
onset of action and short duration of action, which causes rapid alternations of dopamine release in the nucleus accumbens (NA) via disinhibition of dopaminergic neurons in the VTA that project to the NA; a similar method to how opioids, GHB and cannabinoids can modulate dopamine release and reward [20]. Recent clinical study has demonstrated that the administration of alprazolam to human subjects increases blood flow to the NA when analyzed by fMRI [21].

In our current study, we investigated the ability for alprazolam to induce a positive conditioned placed preference in adult female rats. In a previous study performed by our lab, Moffett et al. demonstrated that repeated dosing of alprazolam, a short-acting benzodiazepine could induce strong positive conditioned place preference in male rats [19]. In contrast to our male model, we failed to demonstrate a statistically significant positive CPP in the female rats $(p=0.08$, Figure 4). Despite not achieving significance, the alprazolam group was trending toward a positive CPP effect and had a greater percentage of animals demonstrating positive CPP compared to vehicle. This could be due to a lower number of animals in the female groups compared to the male study (9 vs. 20), and it is likely with a higher group number, statistical significance would be achieved. This needs to be investigated further as well in order to determine if there is a sex difference in rats that influences benzodiazepine-induced reward. There have been some small human studies utilizing fMRI and radiotracing studies that have demonstrated there are increased $\mathrm{GABA}_{\mathrm{A}}$ receptors in females compared to males, but the strength of this evidence is small and requires further studies [22].

There remains a paucity of studies on mechanisms that may regulate benzodiazepine reward in both humans and animals. It is known that $\mathrm{GABA}_{\mathrm{A}}$ receptors on interneurons that regulate dopamine release in the VTA are modulated via benzodiazepines through disinhibition in electrophysiological models. Additionally, it has been demonstrated by Spence et al. that utilizing the $\mathrm{GABA}_{\mathrm{A}}$ benzodiazepine binding site antagonist flumazenil reverses fully the self-administration behaviors of rats for both methamphetamine by rats [23]. Additionally, both flumazenil and the translocator protein (TSPO) antagonist, PK11195, partially reversed the rewarding effects of oxazepam (a structurally and pharmacokinetically similar benzodiazepine to alprazolam); when concurrently administered, these completely abolished the benzodiazepine reward. This suggests a role for both the $\mathrm{GABA}_{\mathrm{A}}$ and TSPO receptors in benzodiazepine-induced reward. Future studies will incorporate the utilization of the $\mathrm{GABA}_{\mathrm{A}}$ and TSPO antagonists in the modulation of the reward response for both male and female rats as well as the roles of these receptors in modulating withdrawal behaviors following chronic administration of benzodiazepines.

\section{Acknowledgements}

The study was funded by an unrestricted grant from The Alliance for Benzodia- 
zepine Best Practices: https://benzoreform.org/.

\section{Conflicts of Interest}

The authors declare no conflicts of interest regarding the publication of this paper.

\section{References}

[1] López-Muñoz, F., Álamo, C. and García-García, P. (2011) The Discovery of Chlordiazepoxide and the Clinical Introduction of Benzodiazepines: Half a Century of Anxiolytic Drugs. Journal of Anxiety Disorders, 25, 554-562. https://doi.org/10.1016/j.janxdis.2011.01.002

[2] Wick, J.Y. (2013) The History of Benzodiazepines. The Consultant Pharmacist, 28, 538-548. https://doi.org/10.4140/TCP.n.2013.538

[3] Griffin III, C.E., Kaye, A.M., Bueno, F.R. and Kaye, A.D. (2013) Benzodiazepine Pharmacology and Central Nervous System-Mediated Effects. Ochsner Journal, 13, 214-223.

[4] Schmitz, A. (2016) Benzodiazepine Use, Misuse, and Abuse: A Review. Mental Health Clinician, 6, 120-126. https://doi.org/10.9740/mhc.2016.05.120

[5] Agarwal, S.D. and Landon, B.E. (2019) Patterns in Outpatient Benzodiazepine Prescribing in the United States. JAMA Network Open, 2, e187399. https://doi.org/10.1001/jamanetworkopen.2018.7399

[6] Guina, J. and Merrill, B. (2018) Benzodiazepines I: Upping the Care on Downers: The Evidence of Risks, Benefits and Alternatives. Journal of Clinical Medicine, 7, Article ID: 17. https://doi.org/10.3390/jcm7020017

[7] Ashman, J.J., Schappert, S.M. and Santo, L. (2020) Emergency Department Visits among Adults Aged 60 and Over: United States, 2014-2017. National Center for Health Statistics Data Brief, 367, 1-8.

[8] Santo, L., Rui, P. and Ashman, J.J. (2020) Physician Office Visits at Which Benzodiazepines Were Prescribed: Findings from 2014-2016 National Ambulatory Medical Care Survey. National Health Statistics Reports, 137, 1-16.

[9] Rui, P., Santo, L. and Ashman, J.J. (2020) Trends in Opioids Prescribed at Discharge from Emergency Departments among Adults: United States, 2006-2017. National Health Statistics Reports, 135, 1-12.

[10] Longo, L.P. and Johnson, B. (2000) Addiction: Part I. Benzodiazepines-Side Effects, Abuse Risk and Alternatives. American Family Physician, 61, 2121-2128.

[11] Ashton, H. (1991) Protracted Withdrawal Syndromes from Benzodiazepines. Journal of Substance Abuse Treatment, 8, 19-28. https://doi.org/10.1016/0740-5472(91)90023-4

[12] Volkow, N.D., et al. (2010) Addiction: Decreased Reward Sensitivity and Increased Expectation Sensitivity Conspire to Overwhelm the Brain's Control Circuit. Bioessays, 32, 748-755. https://doi.org/10.1002/bies.201000042

[13] White, N.M. (2011) Reward: What Is It? How Can It Be Controlled from Behavior? In: Gottfried, J., Ed., Neurobiology of Sensation and Reward, CRC Press/Taylor \& Francis, Boca Raton, 45-60.

[14] Prus, A.J., James, J.R. and Rosecrans J.A. (2009) Conditioned Place Preferance. In: Buccafusco, J.J., Ed., Methods of Behavior Analysis in Neuroscience, CRC Press/Taylor \& Francis, Boca Raton, 59-76. 
[15] McHugh, R.K., et al. (2021) Sex Differences in Benzodiazepine Misuse among Adults with Substance Use Disorders. Addictive Behaviors, 112, Article ID: 106608. https://doi.org/10.1016/j.addbeh.2020.106608

[16] Russo, S.J., et al. (2003) Gonadal Hormones Differentially Modulate Cocaine-Induced Conditioned Place Preference in Male and Female Rats. Neuroscience, 120, 523-533. https://doi.org/10.1016/S0306-4522(03)00317-8

[17] Wang, D.M., Zhang, J.J., Huang, Y.B., Zhao, Y.Z. and Sui, N. (2019) Peripubertal Stress of Male, But Not Female Rats Increases Morphine-Induced Conditioned Place Preference and Locomotion in Adulthood. Development Psychobiology, 61, 920-929. https://doi.org/10.1002/dev.21839

[18] Hilderbrand, E.R. and Lasek, A.W. (2014) Sex Differences in Cocaine Conditioned Place Preference in C57BL/6J Mice. NeuroReport, 25, 105-109.

https://doi.org/10.1097/WNR.0000000000000053

[19] Moffett, C., et al. (2021) The FDA Mandate to Reassess Benzodiazepines: Alprazolam Induces a Positive Conditioned Place-Preference in Male Rats. Journal of Biosciences and Medicines, 9, 1-8. https://doi.org/10.4236/jbm.2021.93001

[20] Tan, K.R., et al. (2010) Neural Bases for Addictive Properties of Benzodiazepines. Nature, 463, 769-774. https://doi.org/10.1038/nature08758

[21] Wolf, D.H., et al. (2013) Oral Alprazolam Acutely Increases Nucleus Accumbens Perfusion. Molecular Psychiatry, 18, 960-961. https://doi.org/10.1038/mp.2012.139

[22] Esterlis, I., et al. (2013) Sex-Specific Differences in GABA - -Benzodiazepine Receptor Availability: Relationship with Sensitivity to Pain and Tobacco Smoking Craving. Addiction Biology, 18, 370-378. https://doi.org/10.1111/j.1369-1600.2011.00403.x

[23] Spence, A.L., Guerin, G.F. and Goeders, N.E. (2016) The Differential Effects of Alprazolam and Oxazepam on Methamphetamine Self-Administration in Rats. Drug and Alcohol Dependence, 166, 209-217. https://doi.org/10.1016/j.drugalcdep.2016.07.015 